\title{
Asymptotic expansion for closed orbits in homology classes for Anosov flows
}

\author{
BY DONGSHENG LIU \\ Department of Physics, Lancaster University, Lancaster, \\ $L A 14 Y B$ \\ e-mail: d.liu@lancaster.ac.uk \\ (Received 2 April 2002)
}

\section{Abstract}

In this paper we give an asymptotic expansion including error terms for the number of closed orbits in the homology classes for homological full Anosov flows. In particular we obtain formulae concerning the coefficients of error terms which depend on the homology classes.

\section{Introduction}

Let $M$ be a $C^{\infty}$ compact manifold. We call a $C^{1}$ flow $\phi_{t}: M \rightarrow M$ an Anosov flow if the tangent bundle $T M$ has a continuous splitting

$$
T M=E^{0} \oplus E^{u} \oplus E^{s}
$$

into $D \phi_{t}$-invariant subbundles such that:

(1) $E^{0}$ is the one dimensional bundle tangent to the flow;

(2) there exist $C, \lambda>0$ such that

$$
\begin{array}{ll}
\left\|D \phi_{t} \mid E^{s}\right\| \leqslant C e^{-\lambda t} & \text { for } t \geqslant 0, \\
\left\|D \phi_{-t} \mid E^{u}\right\| \leqslant C e^{-\lambda t} & \text { for } t \geqslant 0 .
\end{array}
$$

We say that $\phi$ is transitive if there is a dense orbit. We shall restrict attention to transitive flows. We can model an Anosov flow by a suspended flow over a shift of finite type [2].

We assume that $M$ is a $C^{\infty}$ compact manifold with first Betti number $b>0$. For simplicity, we assume that the first homology group of the manifold $M$ is torsion free. We know that there is a isomorphism between $H_{1}(M, \mathbb{Z})$ and $\mathbb{Z}^{b}$. So we can identify the homology group $H_{1}(M, \mathbb{Z})$ with $\mathbb{Z}^{b}$. There are $C^{1}(M)$ functions $F=\left(F_{1}, \ldots, F_{b}\right)$. For a closed orbit $\gamma$ of $\phi, \int_{\gamma} F=\left(\int_{\gamma} F_{1}, \ldots, \int_{\gamma} F_{b}\right)$ represents the homology class of $\gamma$, say, $[\gamma] \in H_{1}(M, \mathbb{Z})$. Let $M_{\phi}$ be the set of $\phi$-invariant probability measures on $M$. For $u \in \mathbb{R}^{b}$, we define the function $\beta(u): \mathbb{R}^{b} \rightarrow \mathbb{R}$ by

$$
\beta(u)=P(\langle u, F\rangle)=\sup _{m \in M_{\phi}}\left\{h_{m}+\left\langle u, \int F d m\right\rangle\right\} .
$$

$\beta(u)$ is analytic and strictly convex on $\mathbb{R}^{b}$. We say that an Anosov flow $\phi$ is homologically full if every homology class contains a closed orbit. If $\phi$ is homologically 
full then it is topologically weak mixing. The function $\beta$ is bounded below and there exists a unique $\xi \in \mathbb{R}^{b}$ for which the infimum is attained [11].

It is easy to see that $\nabla \beta(\xi)=0$. Let $m_{\langle u, F\rangle}$ be the equilibrium state of $\langle u, F\rangle$ and $h^{*}=\beta(\xi)$. Since

$$
\nabla \beta(u)=\int F d m_{\langle u, F\rangle},
$$

we have

$$
h^{*}=\sup \left\{h_{m}: \int F d m=0, m \in M_{\phi}\right\} .
$$

It is well known that $\beta(u)$ can be continued analytically in a neighbourhood of $\xi$ in $\mathbb{C}^{b}$.

Let $\Gamma$ be the set of closed orbits for Anosov flow $\phi$. When $\gamma \in \Gamma$, let $l(\gamma)$ denote the length of $\gamma$, i.e, $l(\gamma)=\int_{\gamma} 1$. If $\phi$ is a homologically full transitive Anosov flow, for $\alpha \in H_{1}(M, \mathbb{Z})$ Sharp [11] obtained

$$
\pi(T, \alpha):=\#\{\gamma \in \Gamma, l(\gamma) \leqslant T,[\gamma]=\alpha\} \sim c e^{\left\langle\xi, \alpha^{\prime}\right\rangle} \frac{e^{T h^{*}}}{T^{b / 2+1}} \quad \text { as } T \longrightarrow \infty,
$$

where $\xi, h^{*}$ are the constants we mentioned above and $\alpha^{\prime}$ is the torsion-free part of $\alpha$.

In [8], Pollicott and Sharp used the results of Dolgopyat's work ([3]) on Anosov flows to obtain a more detailed expansion for $\pi(T, \alpha)$ when $\phi$ is a homologically full transitive Anosov flow. That is, there exists $\delta>0$, such that for $N=[2 \delta]$, there exist $c_{1}, c_{2}, \ldots, c_{N}$ such that

$$
\pi(T, \alpha)=\frac{e^{T h^{*}}}{T^{b / 2+1}}\left(\sum_{n=0}^{N} \frac{c_{n}}{T^{n / 2}}+O\left(T^{-\delta}\right)\right) \quad \text { as } T \longrightarrow \infty .
$$

In this paper, we shall see that if $n$ is odd, then $c_{n}=0$. We also give formulae for $c_{n}$ to describe how the $c_{n}$ depend on the homology class $\alpha$. The main result is the following.

TheORem. Let $M$ be a compact manifold with Betti number $b>0$ and let $\phi_{t}: M \rightarrow M$ be a homologically full transitive Anosov flow. There exist $\xi \in \mathbb{R}^{b}, h^{*}>0$ and $\delta>0$ such that for $\alpha \in H_{1}(M, \mathbb{Z}) \cong \mathbb{Z}^{b}$, we have

$$
\pi(T, \alpha)=\frac{e^{T h^{*}}}{T^{b / 2+1}} e^{-\langle\xi, \alpha\rangle}\left(c_{0}+\sum_{n=1}^{N} \frac{c_{n}(\alpha)}{T^{n}}+O\left(\frac{1}{T^{\delta}}\right)\right) \quad \text { as } T \longrightarrow \infty,
$$

for $N<\delta$ where $c_{0}>0$ is a constant which is independent of $\alpha$. If we write $\alpha=$ $\left(\alpha_{1}, \alpha_{2}, \ldots, \alpha_{b}\right)$ then the constants $c_{n}(\alpha)$ are in the form

$$
c_{n}(\alpha)=\sum_{l_{1}+l_{2}+\cdots+l_{b}=0}^{2 n} c_{l_{1} l_{2} \cdots l_{d}} \alpha_{1}^{l_{1}} \alpha_{2}^{l_{2}} \cdots \alpha_{b}^{l_{b}},
$$

where $c_{l_{1}, l_{2}, \ldots, l_{b}}$ are constants which are independent of $\alpha$.

The analysis in this paper is closely akin to that used by Anantharaman in [1]. but we give more details of the proofs and more explicit information, necessary to 
understand the role of the homology class $\alpha$. In the last section we use an approximation argument to determine how the coefficients depend on the homology classes.

\section{Counting function}

We assume that $g$ has compact support and has $C^{\infty}$-regularity. Let $g \geqslant 0$. For $\alpha \in H_{1}(M, \mathbb{Z})$, we define the auxiliary function

$$
\pi_{g}(T, \alpha)=\sum_{\gamma \in \Gamma,[\gamma]=\alpha} g(l(\gamma-T))
$$

Let $\hat{g}$ be the Fourier transform of $g$, By Fourier's inverse transform formula,

$$
\begin{aligned}
\pi_{g}(T, \alpha) & =\sum_{[\gamma]=\alpha} g(l(\gamma)-T) e^{\sigma(l(\gamma)-T)} e^{\sigma T} e^{-\sigma l(\gamma)} \\
& =\frac{1}{2 \pi} \sum_{\gamma \in \Gamma} \int_{\mathbb{R}} \int_{\mathbb{R}^{b} / \mathbb{Z}^{b}} \hat{g}(-i \sigma+t) e^{-i t(l(\gamma)-T)} e^{\sigma T} e^{-\sigma l(\gamma)} e^{\langle\xi+2 \pi i v,[\gamma]\rangle} e^{-\langle\xi+2 \pi i v, \alpha\rangle} d v d t \\
& =\frac{1}{2 \pi} \int_{\mathbb{R}} \int_{\mathbb{R}^{b} / \mathbb{Z}^{b}} Z(\sigma+i t, v) e^{\sigma T+i t T} \hat{g}(-i \sigma+t) e^{-\langle\xi+2 \pi i v, \alpha\rangle} d v d t
\end{aligned}
$$

where we have defined

$$
Z(s, v)=Z(\sigma+i t, v)=\sum_{\gamma \in \Gamma} e^{-s l(\gamma)+\langle\xi+2 \pi i v,[\gamma]\rangle}=\sum_{\gamma \in \Gamma} e^{-s l(\gamma)+\left\langle\xi+2 \pi i v, \int_{\gamma} F\right\rangle}
$$

for $(s, v) \in \mathbb{C} \times \mathbb{R}^{b} / \mathbb{Z}^{b}$. It is well known that when $\operatorname{Res}=\sigma>\beta(\xi)=h^{*}, Z(s, v)$ is absolutely convergent.

For the behaviour of $Z(s, v)$ in the neighbourhood of $R e s=h^{*}$, we have the following proposition.

Proposition 1. There exist $B>0, c>0, \epsilon>0, \beta>0, \rho>0$ and an open set $V_{0}, a$ neighbourhood of 0 in $\mathbb{R}^{d} / \mathbb{Z}^{d}$, such that:

(1) $Z(s, v)$ is analytic in $\left\{s=\sigma+i t: \sigma>h^{*}-\frac{c}{|t|^{\rho}},|t|>B\right\}$, and in this domain $|Z(s, v)|=O\left(|t|^{\beta}\right)$

(2) $Z(s, v)+\log (s-\beta(\xi+i v))$ is analytic in $\left\{(s, v): v \in V_{0}, \sigma>h^{*}-\epsilon,|t| \leqslant B\right\}$;

(3) $Z(s, v)$ is analytic in $\left\{(s, v): v \notin \bar{V}_{0}, \sigma>h-\epsilon,|t| \leqslant B\right\}$.

Proof. The proof of part (1) is analogous to [8]. We refer to [1] for the proof of (2) and (3). For details see [5].

Now in order to estimate $\pi_{g}(T, \alpha)$, we only need to calculate the integral

$$
\frac{1}{2 \pi} \int_{\mathbb{R} \times \mathbb{R}^{b} / \mathbb{Z}^{b}} Z(\sigma+i t, v) e^{(\sigma+i t) T} \hat{g}(-i \sigma+t) e^{-\langle\xi+2 \pi i v, \alpha\rangle} d t d v .
$$

In the following let $g$ be of class $C^{\infty}$ with compact support and $\sigma>h^{*}$. We will divide $\mathbb{R}^{b} / \mathbb{Z}^{b}$ into $V_{0}$ and $\mathbb{R}^{b} / \mathbb{Z}^{b}-V_{0}$. Then

$$
\begin{aligned}
\pi_{g}(T, \alpha)= & \frac{1}{2 \pi} \int_{\mathbb{R} \times \mathbb{R}^{b} / \mathbb{Z}^{b}} Z(\sigma+i t, v) e^{(\sigma+i t) T} \hat{g}(-i \sigma+t) e^{-\langle\xi+2 \pi i v, \alpha\rangle} d t d v \\
= & \left.\frac{1}{2 \pi} \int_{V_{0}} e^{-\langle\xi+2 \pi i v, \alpha\rangle} d v \int_{\mathbb{R}} Z(\sigma+i t, v) e^{(\sigma+i t) T} \hat{g}(-i \sigma+t)\right) d t \\
& \left.+\frac{1}{2 \pi} \int_{\mathbb{R}^{b} / \mathbb{Z}^{b}-V_{0}} e^{-\langle\xi+2 \pi i v, \alpha\rangle} d v \int_{\mathbb{R}} Z(\sigma+i t, v) e^{(\sigma+i t) T} \hat{g}(-i \sigma+t)\right) d t
\end{aligned}
$$


We shall consider the two integrals separately. First we consider the integral over $\mathbb{R}^{b} / \mathbb{Z}^{b}-V_{0}$. For $v \notin V_{0}$, we have the following estimate.

Lemma 1. For $v \notin V_{0}$ and for all $\sigma>h^{*}$,

$$
\left|\int_{\mathbb{R}} Z(\sigma+i t, v) e^{(\sigma+i t) T} \hat{g}(-i \sigma+t) d t\right|=O\left(\left\|g^{(m)}\right\|_{L^{1}} e^{h^{*} T} / T^{(m-\beta) K}\right),
$$

for any $K, m \in \mathbb{N}$.

Proof. By Proposition 1 and Cauchy's Theorem

$$
\int_{\Delta} Z(s, v) e^{s T} \hat{g}(-i s) d s=0
$$

where $\Delta=\{$ Res $=\sigma, \mid$ Ims $\mid \leqslant R\} \cup\{\operatorname{Res}=c(R), \mid$ Ims $\mid \leqslant R\} \cup\{c(R) \leqslant$ Res $\leqslant \sigma, \mid$ Ims $\mid=$ $R\}$, and where $c(R)=h^{*}-c / R^{\rho}, R=T^{K}$ for some $K$, and $\sigma=h^{*}+1 / T$. Since $g$ is of class $C^{\infty}$ with compact support, for any $m \in \mathbb{N}$,

$$
|\hat{g}(-i \sigma+t)| \leqslant c\left\|g^{(m)}\right\|_{L^{1}} /|t|^{m} .
$$

So

$$
\begin{aligned}
& \left|\int_{\mathbb{R}} Z(\sigma+i t, v) e^{(\sigma+i t) T} \hat{g}(-i \sigma+t) d t-\int_{-R}^{R} Z(\sigma+i t, v) e^{(\sigma+i t) T} \hat{g}(-i \sigma+t) d t\right| \\
& \quad \leqslant \int_{T^{K}}^{\infty} e^{\sigma T} c|t|^{\beta} c \frac{\left\|g^{(m)}\right\|_{L^{1}}}{|t|^{m}} d t=O\left(\frac{\left\|g^{(m)}\right\|_{L^{1}} e^{h^{*} T}}{T^{(m-1-\beta) K}}\right) .
\end{aligned}
$$

On the other hand,

$$
\left|\int_{R e s=c(R),|I m s| \leqslant R} Z(s, v) e^{s T} \hat{g}(-i s) d s\right|=O\left(\frac{\left\|g^{(m)}\right\|_{L^{1}}}{T^{(m-1-\beta) K}} e^{h^{*} T} e^{-\frac{c T}{T^{K} \rho}}\right),
$$

and

$$
\left|\int_{c(R) \leqslant R e s \leqslant \sigma,|I m s|=R} Z(s, v) e^{s T} \hat{g}(-i s) d s\right|=O\left(\left\|g^{(m)}\right\|_{L^{1}} \frac{e^{h^{*} T}}{T^{(m-\beta) K}}\right) .
$$

Since $c T / T^{K \rho}>0$, this completes the proof.

Now we consider the integral over $V_{0}$. We shall prove the following lemma.

Lemma 2. Let $v \in V_{0}$; then for all $M \in \mathbb{N}$ and for all $\sigma>h^{*}$, we have

$$
\begin{aligned}
& \left|\int_{\mathbb{R}} Z(\sigma+i t, v) e^{(\sigma+i t) T} \hat{g}(-i \sigma+t) d t-2 \pi \sum_{j=0}^{M} \frac{1}{T^{j+1}} \frac{d^{j} \hat{g}}{d s^{j}}(-i \beta(\xi+i v)) e^{\beta(\xi+i v) T}\right| \\
& \leqslant c\left(\|g\|_{L^{1}}+\cdots+\left\|y^{M} g\right\|_{L^{1}}\right) e^{T h^{*}-\frac{c T}{T^{K} \rho}}+\left\|g^{(m)}\right\|_{L^{1}} e^{T h^{*}}\left(\frac{c_{1}}{T^{(m-1-\beta) K}}+\frac{c_{2}}{T^{(m-\beta) K}}\right) \\
& \quad+\frac{c}{T^{M+1}}\left|\int_{C(\sigma)} \log (s-\beta(\xi+i v)) e^{s T} \frac{d^{M+1}}{d s^{M+1}} \hat{g}(-i s) d s\right|
\end{aligned}
$$

where $C(\sigma)$ will be defined later.

Proof. When $v \in V_{0}, \sigma$ is fixed with $\sigma>h^{*}$. For $2 B<R \in \mathbb{R}^{+}$, let $C_{0}=\{$ Res $=c(R),-2 B \leqslant I m s \leqslant 2 B\} ; C_{1}=\{$ Ims $=-2 B, c(R) \leqslant$ Res $\leqslant \sigma\} ;$

$C_{2}=\{$ Res $=\sigma,-2 B \leqslant I m s \leqslant 2 B\} ; C_{3}=\{\operatorname{Ims}=2 B, c(R) \leqslant \operatorname{Res} \leqslant \sigma\} ;$ 
$C_{4}^{+}=\{$Res $=c(R), 2 B \leqslant I m s \leqslant R\} ; C_{4}^{-}=\{\operatorname{Res}=c(R),-R \leqslant I m s \leqslant-2 B\} ;$

$C_{5}^{+}=\{$Res $=\sigma, 2 B \leqslant I m s \leqslant R\} ; C_{5}^{-}=\{$Res $=\sigma,-R \leqslant I m s \leqslant-2 B\} ;$

$C_{R}^{+}=\{c(R) \leqslant R e s \leqslant \sigma$, Ims $=R\} ; C_{R}^{-}=\{c(R) \leqslant$ Res $\leqslant \sigma$, Ims $=-R\}$.

By part (1) of Proposition 1, Z(s,v) is analytic in Res $\geqslant c(R),|\operatorname{Im} s|>B$, so

$$
\int_{\left\{C_{3} \cup C_{5}^{+} \cup C_{R}^{+} \cup C_{4}^{+}\right\}} Z(s, v) e^{s T} \hat{g}(-i s) d s=0
$$

and

$$
\int_{\left\{C_{1} \cup C_{4}^{-} \cup C_{R}^{-} \cup C_{5}^{-}\right\}} Z(s, v) e^{s T} \hat{g}(-i s) d s=0 .
$$

By part (2) of Proposition 1, we have

$$
\int_{-\left\{C_{0} \cup C_{3} \cup C_{2} \cup C_{1}\right\}}(Z(s, v)+\log (s-\beta(\xi+i v))) e^{s T} \hat{g}(-i s) d s=0,
$$

where the three contours are counterclockwise and $-C_{i}$ means that the orientation of the path is reversed.

From (1), we have

$$
\int_{C_{5}^{+}} Z(s, v) e^{s T} \hat{g}(-i s) d s=\int_{-\left\{C_{3} \cup C_{4}^{+} \cup C_{R}^{+}\right\}} Z(s, v) e^{s T} \hat{g}(-i s) d s .
$$

From (2), we have

$$
\int_{C_{5}^{-}} Z(s, v) e^{s T} \hat{g}(-i s) d s=\int_{-\left\{C_{1} \cup C_{4}^{-} \cup C_{R}^{-}\right\}} Z(s, v) e^{s T} \hat{g}(-i s) d s .
$$

From (3), we have

$$
\begin{aligned}
& \int_{-C_{2}} Z(s, v) e^{s T} \hat{g}(-i s) d s=\int_{C_{1} \cup C_{2} \cup C_{3}} \log (s-\beta(\xi+i v)) e^{s T} \hat{g}(-i s) d s \\
& +\int_{C_{0}}(Z(s, v)+\log (s-\beta(\xi+i v))) e^{s T} \hat{g}(-i s) d s+\int_{C_{1} \cup C_{3}} Z(s, v) e^{s T} \hat{g}(-i s) d s .
\end{aligned}
$$

Let $C(\sigma)=C_{1} \cup C_{2} \cup C_{3}$. Adding the three identities (4), (5), (6) we obtain

$$
\begin{aligned}
& \int_{\mathbb{R}} Z(\sigma+i t, v) e^{(\sigma+i t) T} \hat{g}(-i \sigma+t) d t \\
& =-i \int_{C(\sigma)} \log (s-\beta(\xi+i v)) \hat{g}(-i s) e^{s T} d s+i \int_{\left\{C_{4}^{+} \cup C_{4}^{-}\right\}} Z(s, v) e^{s T} \hat{g}(-i s) d s \\
& \quad-i \int_{C_{0}}(Z(s, v)+\log (s-\beta(\xi+i v))) e^{s T} \hat{g}(-i s) d s+i \int_{C_{R}^{+} \cup C_{R}^{-}} Z(s, v) e^{s T} \hat{g}(-i s) d s \\
& \quad+\left(\int_{R}^{\infty}+\int_{-\infty}^{-R}\right) Z(\sigma+i t, v) e^{(\sigma+i t) T} \hat{g}(-i \sigma+t) d t
\end{aligned}
$$


As we discussed in Lemma 1 , for any $m, K \in \mathbb{N}$,

$$
\begin{gathered}
\int_{\left\{C_{R}^{+} \cup C_{R}^{-}\right\}} Z(s, v) e^{s T} \hat{g}(-i s) d s \leqslant c_{1}\left\|g^{(m)}\right\|_{L^{1}} \frac{e^{h^{*} T}}{T^{(m-\beta) K}} \\
\left(\int_{-\infty}^{-R}+\int_{R}^{\infty}\right) Z(\sigma+i t, v) e^{(\sigma+i t) T} \hat{g}(-i \sigma+t) d s \leqslant c_{2}\left\|g^{(m)}\right\|_{L^{1}} \frac{e^{h^{*} T}}{T^{(m-1-\beta) K}} \\
\left.\int_{C_{0}}(Z(s, v)+\log (s-\beta(\xi+i v))) e^{s T} \hat{g}(-i s) d s=c_{3}\|g\|_{L^{1}} e^{h^{*} T-\frac{c T}{T^{K} \rho}}\right)
\end{gathered}
$$

and

$$
\int_{\left\{C_{4}^{+} \cup C_{4}^{-}\right\}} Z(s, v) e^{s T} \hat{g}(-i s) d s \leqslant c_{4} \frac{\left\|g^{(m)}\right\|_{L^{1}}}{T^{(m-1-\beta) K}} e^{h^{*} T} e^{-\frac{c T}{T^{K} \rho}} \leqslant c_{4} \frac{\left\|g^{(m)}\right\|_{L^{1}}}{T^{(m-1-\beta) K}} .
$$

Hence

$$
\begin{aligned}
& \left|\int_{\mathbb{R}} Z(\sigma+i t, v) e^{(\sigma+i t) T} \hat{g}(-i \sigma+t) d t+i \int_{C(\sigma)} \log (s-\beta(\xi+i v)) \hat{g}(-i s) e^{s T} d s\right| \\
& \quad \leqslant e^{T h^{*}}\left(\frac{c_{1}\left\|g^{(m)}\right\|_{L^{1}}}{T^{(m-1-\beta) K}}+\frac{c_{2}\left\|g^{(m)}\right\|_{L^{1}}}{T^{(m-\beta) K}}+c_{3}\|g\|_{L^{1}} e^{-\frac{c T}{T^{K} \rho}}\right) .
\end{aligned}
$$

Next we consider $i \int_{C(\sigma)} \log (s-\beta(\xi+i v)) \hat{g}(-i s) e^{s T} d s$. Integrating by parts, we have

$$
\begin{aligned}
& \mid i \int_{C(\sigma)} \log (s-\beta(\xi+i v)) \hat{g}(-i s) e^{s T} d s+\frac{i}{T} \int_{C(\sigma)} \frac{\hat{g}(-i s)}{s-\beta(\xi+i v)} e^{s T} d s \\
& \quad+\frac{i}{T} \int_{C(\sigma)} \log (s-\beta(\xi+i v)) \frac{d \hat{g}}{d s}(-i s) e^{s T} d s \mid \\
& \quad=\left|\frac{i}{T} \log (s-\beta(\xi+i v)) \hat{g}(-i s) e^{s T}\right|_{s=c(R)+2 B i}^{s=c(R)-2 B i} \leqslant c\|g\|_{L^{1}} e^{T h^{*}-\frac{c T}{T^{K} \rho}} .
\end{aligned}
$$

For the integral $\int_{C(\sigma)} \frac{\hat{g}(-i s)}{s-\beta(\xi+i v)} e^{s T} d s$, we use the residue formula,

$$
\int_{C(\sigma) \cup C_{0}} \frac{\hat{g}(-i s)}{s-\beta(\xi+i v)} e^{s T} d s=-2 \pi i \hat{g}(-i \beta(\xi+i v)) e^{\beta(\xi+i v) T} .
$$

So

$$
\begin{aligned}
& \left|\int_{C(\sigma)} \frac{\hat{g}(-i s)}{s-\beta(\xi+i v)} e^{s T} d s+2 \pi i \hat{g}(-i \beta(i v)) e^{\beta(\xi+i v) T}\right| \\
& =\left|\int_{C_{0}} \frac{\hat{g}(-i s)}{s-\beta(\xi+i v)} e^{s T} d s\right| \leqslant c\|g\|_{L^{1}} e^{T h^{*}-\frac{c T}{T^{K} \rho}} .
\end{aligned}
$$

Now we have obtained

$$
\begin{aligned}
& \left|\int_{\mathbb{R}} Z(\sigma+i t, v) e^{(\sigma+i t) T} \hat{g}(-i \sigma+t) d t-2 \pi \frac{1}{T} \hat{g}(-i \beta(\xi+i v)) e^{\beta(i v) T}\right| \\
& \leqslant e^{T h^{*}}\left(\frac{c_{1}\left\|g^{(m)}\right\|_{L^{1}}}{T^{(m-1-\beta) K}}+\frac{c_{2}\left\|g^{(m)}\right\|_{L^{1}}}{T^{(m-\beta) K}}+c_{3}\|g\|_{L^{1}} e^{-\frac{c T}{T^{K} \rho}}\right) \\
& +\frac{c}{T}\left|\int_{C(\sigma)} \log (s-\beta(\xi+i v)) e^{s T} \frac{d \hat{g}(-i s)}{d s} d s\right|
\end{aligned}
$$


We iterate the preceding operation $M+1$ times, and note that

$$
\left|\frac{d^{k} \hat{g}(-i s)}{d s^{k}}\right|=\left|\int_{\mathbb{R}} y^{k} g(y) e^{s y} d y\right| \leqslant c\left\|y^{k} g\right\|_{L^{1}} .
$$

We have

$$
\begin{aligned}
& \left|\int_{\mathbb{R}} Z(\sigma+i t, v) e^{(\sigma+i t) T} \hat{g}(-i \sigma+t) d t-2 \pi \sum_{j=0}^{M} \frac{1}{T^{j+1}} \frac{d^{j} \hat{g}}{d s^{j}}(-i \beta(\xi+i v)) e^{\beta(\xi+i v) T}\right| \\
& \leqslant c_{1}^{\prime}\left\|g^{(m)}\right\|_{L^{1}} e^{T h^{*}}\left(\frac{c_{1}}{T^{(m-1-\beta) K}}+\frac{c_{2}}{T^{(m-\beta) K}}\right)+c_{2}^{\prime}\left(\|g\|_{L^{1}}+\cdots+\left\|y^{M} g\right\|_{L^{1}}\right) e^{T h^{*}-\frac{c T}{T^{K}}} \\
& \quad+\frac{c}{T^{M+1}}\left|\int_{C(\sigma)} \log (s-\beta(\xi+i v)) e^{s T} \frac{d^{M+1}}{d s^{M+1}} \hat{g}(-i s) d s\right|
\end{aligned}
$$

Since

$$
\begin{aligned}
\lim _{\sigma \rightarrow h^{*}} \int_{V_{0}} e^{-2 \pi i\langle v, \alpha\rangle} d v \int_{C(\sigma)} \log (s-\beta(i v)) e^{s T} \frac{d^{M+1}}{d s^{M+1}} \hat{g}(-i s) d s \\
\quad=\int_{V_{0}} e^{-2 \pi i\langle v, \alpha\rangle} d v \int_{C\left(h^{*}\right)} \log (s-\beta(i v)) e^{s T} \frac{d^{M+1}}{d s^{M+1}} \hat{g}(-i s) d s \\
\quad=O\left(\frac{\left\|y^{M+1} g\right\|_{L^{1}}}{T^{M+1}} e^{T h^{*}}\right),
\end{aligned}
$$

by Lemma 1 and Lemma 2, we can prove the following proposition.

Proposition 2. Let $g$ be class $C^{\infty}$ with compact support. For all $M, m \geqslant 1$, we have

$$
\begin{aligned}
& \left|\pi_{g}(T, \alpha)-e^{-\langle\xi, \alpha\rangle} \sum_{j=0}^{M} \frac{1}{T^{j+1}} \int_{V_{0}} e^{-2 \pi i\langle v, \alpha\rangle} \frac{d^{j} \hat{g}}{d s^{j}}(-i \beta(\xi+i v)) e^{\beta(\xi+i v) T} d v\right| \\
& \leqslant c_{1}^{\prime} \frac{\left\|y^{M+1} g\right\|_{L^{1}}}{T^{M+1}} e^{T h^{*}}+c_{2}^{\prime}\left\|g^{(m)}\right\|_{L^{1}} e^{T h^{*}}\left(\frac{c_{1}}{T^{(m-1-\beta) K}}+\frac{c_{2}}{T^{(m-\beta) K}}\right) \\
& \quad+c_{3}^{\prime}\left(\|g\|_{L^{1}}+\cdots+\left\|y^{M} g\right\|_{L^{1}}\right) e^{T h^{*}-\frac{c T}{T^{K \rho}}}
\end{aligned}
$$

For $\forall N \in \mathbb{N}$, taking $m$ sufficiently so large such that $(m-\beta) K \geqslant N+\frac{b}{2}+2$ and $M=N+b+2$, we have

Proposition 3. Let $g$ be class $C^{\infty}$ with compact support. For all $N \geqslant 1$, we have

$$
\begin{aligned}
& \left|\pi_{g}(T, \alpha)-e^{-\langle\xi, \alpha\rangle} \sum_{j=0}^{N+b+2} \frac{1}{T^{j+1}} \int_{V_{0}} e^{-2 \pi i\langle v, \alpha\rangle} \frac{d^{j} \hat{g}}{d s^{j}}(-i \beta(\xi+i v)) e^{\beta(\xi+i v) T} d v\right| \\
& \leqslant c_{1}^{\prime} \frac{\left\|y^{N+b+2} g\right\|_{L^{1}}}{T^{N+b+2}} e^{T h^{*}}+c_{2}^{\prime} \frac{\left\|g^{(m)}\right\|_{L^{1}}}{T^{N+1+\frac{b}{2}+1}} e^{T h^{*}} \\
& \quad+c_{3}^{\prime}\left(\|g\|_{L^{1}}+\cdots+\left\|y^{N+b+2} g\right\|_{L^{1}}\right) e^{T h^{*}-\frac{c T}{T^{K} \rho}} .
\end{aligned}
$$

3. Coefficients of error terms of $\pi_{g}(T, \alpha)$

From Proposition 3 , in order to estimate $\pi_{g}(T, \alpha)$ we only need to estimate

$$
\int_{V_{0}} \frac{d^{j} \hat{g}}{d s^{j}}(-i \beta(\xi+i v)) e^{T \beta(\xi+i v)} e^{-2 \pi i\langle v, \alpha\rangle} d v .
$$


We shall use the method that Anantharaman used in [1]. We first prove the following lemma.

Lemma 3. There exist polynomials $f_{j}^{(k)}(i v)$ in $i v_{1}, \ldots, i v_{b}$ such that the total exponent of each term has the same parity as $k$ and such that

$$
\begin{gathered}
\mid \int_{V_{0}} \frac{d^{j} \hat{g}}{d s^{j}}(-i \beta(\xi+i v)) e^{T \beta(\xi+i v)-2 \pi i\langle v, \alpha\rangle} d v- \\
-\frac{e^{T h^{*}}}{T^{b / 2}} \sum_{k=0}^{2 N+1} \frac{1}{T^{k / 2}} \int_{\|v\| \leqslant \sqrt{T} \rho} e^{-\frac{1}{2} \beta^{\prime \prime}(\xi)(v, v)-2 \pi i\left\langle\frac{v}{\sqrt{T}}, \alpha\right\rangle} f_{j}^{(k)}(i v) d v \mid \leqslant \\
\quad \leqslant c \sup _{n \leqslant 2 N+2}\left\|y^{j+n} g\right\|_{L^{1}} \frac{e^{T h^{*}}}{T^{N+\frac{b}{2}+1}},
\end{gathered}
$$

for some small $\rho$.

\section{Remark.}

(1) Here $\|\cdot\|$ denotes the 2 -norm, i.e., $\|v\|=\left(\sum_{i=1}^{b} v_{i}^{2}\right)^{1 / 2}$.

(2) The proof is similar to that in [1]. We denote $\left(d^{j} \hat{g} / d s^{j}\right)(-i \beta(\xi+i v))$ by $\bar{g}_{j}(i v)$.

For convenience, let $V_{0}$ be of the form $\left\{v \in \mathbb{R}^{b} / \mathbb{Z}^{b}:\|v\| \leqslant \rho\right\}$.

(3) By the first condition, we mean that $f_{j}^{(k)}(i v)$ can be written in the form

$$
f_{j}^{(k)}(i v)=\sum a_{l_{1}, l_{2}, \ldots, l_{b}}\left(i v_{1}\right)^{l_{1}}\left(i v_{2}\right)^{l_{2}} \cdots\left(i v_{b}\right)^{l_{b}} .
$$

In particular,

$$
\begin{aligned}
f_{j}^{(0)}(i v)= & \bar{g}_{j}^{(0)}(0)=\left.\frac{d^{j}}{d s^{j}} \hat{g}(-i s)\right|_{s=h^{*}} \\
f_{j}^{(1)}(i v)= & \frac{1}{6} \bar{g}_{j}^{(0)}(0) \beta^{(3)}(\xi) \cdot(i v)^{3}+\bar{g}_{j}^{(1)}(0) \cdot(i v) ; \\
f_{j}^{(2)}(i v)= & \frac{1}{72} \bar{g}_{j}^{(0)}(0)\left(2\left(\beta^{(3)}(\xi) \cdot(i v)^{3}\right)^{2}+3 \beta^{(4)}(\xi) \cdot(i v)^{4}\right) \\
& +\frac{1}{6} \bar{g}_{j}^{(1)}(0) \cdot(i v) \beta^{(3)}(\xi) \cdot(i v)^{3}+\frac{1}{2} \bar{g}_{j}^{(2)}(0) \cdot(i v)^{2} .
\end{aligned}
$$

(4) We can see that $f_{j}^{(0)}(i v)$ are constants and $f_{0}^{(0)}(i v)>0$, since

$$
\begin{aligned}
f_{j}^{(0)}(i v) & =\left.\frac{d^{j}}{d s^{j}} \hat{g}(-i s)\right|_{s=h^{*}}=\left.\frac{d^{j}}{d s^{j}} \int_{\mathbb{R}} g(y) e^{s y} d y\right|_{s=h^{*}} \\
& =\left.\int_{\mathbb{R}} y^{j} g(y) e^{s y} d y\right|_{s=h^{*}}=\int_{\mathbb{R}} y^{j} g(y) e^{h^{*} y} d y .
\end{aligned}
$$

Next we need to estimate

$$
\sum_{k=0}^{2 N+1} \frac{1}{T^{k / 2}} \int_{\|v\| \leqslant \sqrt{T} \rho} e^{-\frac{1}{2} \beta^{\prime \prime}(\xi)(v, v)} e^{-2 \pi i\langle\alpha, v / \sqrt{T}\rangle} f_{j}^{(k)}(i v) d v .
$$

We have the following proposition. 
Proposition 4.

$$
\begin{aligned}
& \sum_{k=0}^{2 N+1} \frac{1}{T^{k / 2}} \int_{\|v\| \leqslant \sqrt{T} \rho} e^{-\frac{1}{2} \beta^{\prime \prime}(\xi)(v, v)} e^{-2 \pi i\langle\alpha, v / \sqrt{T}\rangle} f_{j}^{(k)}(i v) d v \\
& \quad=\sum_{k=0}^{2 N+1} \frac{1}{T^{k / 2}} \int_{\mathbb{R}^{b}} e^{-\frac{1}{2} \beta^{\prime \prime}(\xi)(v, v)} s_{j}^{(k)}(\alpha, i v) d v+O\left(\sup _{n \leqslant 4 N+b+2}\left\|y^{n} g\right\|_{L^{1}} T^{-(N+1)}\right)
\end{aligned}
$$

where

$$
s_{j}^{(k)}(\alpha, i v)=\sum_{l=0}^{k}(-1)^{k-l} \frac{f_{j}^{(l)}(i v)\langle\alpha, 2 \pi i v\rangle^{k-l}}{(k-l) !}
$$

are polynomials in $i v_{1}, \ldots, i v_{b}$ and we still have that the total exponent of each term in $s_{j}^{(k)}(\alpha, v)$ has the same parity as $k$.

Proof. We expand $e^{-2 \pi i\langle\alpha, v / \sqrt{T}\rangle}$ in a neighbourhood of 0 ,

$$
\begin{aligned}
e^{-2 \pi i\langle\alpha, v / \sqrt{T}\rangle}=1 & -\frac{\langle\alpha, 2 \pi i v\rangle}{\sqrt{T}}+\frac{\langle\alpha, 2 \pi i v\rangle^{2}}{2 T}-\frac{\langle\alpha, 2 \pi i v\rangle^{3}}{3 ! T^{3 / 2}} \\
& +\cdots-\frac{\langle\alpha, 2 \pi i v\rangle^{2 N+1}}{(2 N+1) ! T^{N / 2+1}}+Z_{N}(i v / \sqrt{T}),
\end{aligned}
$$

where $\left|Z_{N}(i v / \sqrt{T})\right| \leqslant c \frac{\|v\|^{2 N+2}}{T^{N+1}}$.

Let

$$
s_{j}^{(k)}(\alpha, i v)=\sum_{l=0}^{k}(-1)^{k-l} \frac{f_{j}^{(l)}(i v)\langle\alpha, 2 \pi i v\rangle^{k-l}}{(k-l) !}
$$

then

$$
\begin{aligned}
& \sum_{k=0}^{2 N+1} \frac{1}{T^{k / 2}} \int_{\|v\| \leqslant \sqrt{T} \rho} e^{-\frac{1}{2} \beta^{\prime \prime}(\xi)(v, v)} e^{-2 \pi i\langle\alpha, v / \sqrt{T}\rangle} f_{j}^{(k)}(i v) d v \\
& \quad=\sum_{k=0}^{2 N+1} \frac{1}{T^{k / 2}} \int_{\|v\| \leqslant \sqrt{T} \rho} e^{-\frac{1}{2} \beta^{\prime \prime}(\xi)(v, v)} s_{j}^{(k)}(\alpha, i v) d v+O\left(\sup _{n \leqslant 4 N+b+2}\left\|y^{n} g\right\|_{L^{1}} T^{-(N+1)}\right) .
\end{aligned}
$$

For $T$ sufficiently large, for all $m \in \mathbb{N}$, we have

$$
\begin{aligned}
& \int_{\|v\|>\sqrt{T} \rho} e^{-\frac{1}{2} \beta^{\prime \prime}(\xi)(v, v)}\|v\|^{m} d v \leqslant \int_{\|v\|>\sqrt{T} \rho} e^{-\epsilon^{\prime}\|v\|^{2}}\left|v_{1} v_{2} \cdot v_{d}\right| d v \\
& \leqslant \prod_{i=1}^{d} \int_{\sqrt{T} \rho}^{\infty} e^{-\epsilon^{\prime} v_{i}^{2}} v_{i} d v_{i} \leqslant c e^{-\epsilon^{\prime} T}
\end{aligned}
$$


for some $\epsilon^{\prime}>0$. So we have

$$
\begin{aligned}
& \mid \sum_{k=0}^{2 N+1} \frac{1}{T^{k / 2}} \int_{\|v\| \leqslant \sqrt{T} \rho} e^{-\frac{1}{2} \beta^{\prime \prime}(\xi)(v, v)} e^{-2 \pi i\langle\alpha, v / \sqrt{T}\rangle} f_{j}^{(k)}(i v) d v \\
& \quad-\sum_{k=0}^{2 N+1} \frac{1}{T^{k / 2}} \int_{\mathbb{R}^{b}} e^{-\frac{1}{2} \beta^{\prime \prime}(\xi)(v, v)} s_{j}^{(k)}(\alpha, i v) d v \mid \\
& \leqslant c \sup _{n \leqslant 4 N+b+2}\left\|y^{n} g\right\|_{L^{1}}\left(T^{-(N+1)}+e^{-\epsilon^{\prime} T}\right) .
\end{aligned}
$$

Now the proof of the proposition is complete.

In the following lemma, we will see that the coefficients of $T^{-\frac{k}{2}}$ vanish.

Lemma 4. If $k$ is odd, then

$$
\int_{\mathbb{R}^{b}} e^{-\frac{1}{2} \beta^{\prime \prime}(\xi)(v, v)} s_{j}^{(k)}(\alpha, i v) d v=0 .
$$

Proof. Since

$$
\begin{aligned}
s_{j}^{(k)}(\alpha, i v) & =\sum_{l=0}^{k}(-1)^{k-l} \frac{f_{j}^{(l)}(i v)\langle\alpha, 2 \pi i v\rangle^{k-l}}{(k-l) !} \\
& =\sum_{l=0}^{k}(-1)^{k-l} f_{j}^{(l)}(i v)(2 \pi i)^{k-l}\left(\sum_{i=1}^{b} \alpha_{i} v_{i}\right)^{k-l} \\
& :=\sum a_{l_{1}, l_{2}, \ldots, l_{b}}(\alpha)\left(i v_{1}\right)^{l_{1}}\left(i v_{2}\right)^{l_{2}} \cdots\left(i v_{b}\right)^{l_{b}}
\end{aligned}
$$

where $l_{1}+l_{2}+\cdots+l_{b}$ is odd and $a_{l_{1}, L_{2}, \ldots, l_{b}}(\alpha)$ are constants which depend on $\alpha$ and $g$. Let $v^{\prime}=-v$, i.e., $\left(v_{1}^{\prime}, v_{2}^{\prime}, \ldots, v_{b}^{\prime}\right)=\left(-v_{1},-v_{2}, \ldots,-v_{b}\right)$, then

$$
\begin{aligned}
\int_{\mathbb{R}^{b}} & e^{-\frac{1}{2} \beta^{\prime \prime}(\xi)(v, v)} s_{j}^{(k)}(\alpha, i v) d v \\
= & \int_{\mathbb{R}^{b}} e^{-\frac{1}{2} \beta^{\prime \prime}(\xi)(v, v)} \sum a_{l_{1}, l_{2}, \ldots, l_{b}}\left(i v_{1}\right)^{l_{1}}\left(i v_{2}\right)^{l_{2}} \cdots\left(i v_{b}\right)^{l_{b}} d v_{1} d v_{2} \cdots d v_{b} \\
= & \int_{\mathbb{R}^{b}} e^{-\frac{1}{2} \beta^{\prime \prime}(\xi)\left(-v^{\prime},-v^{\prime}\right)} \sum a_{l_{1}, l_{2}, \ldots, l_{b}}(-1)^{l_{1}+\cdots+l_{b}}\left(i v_{1}^{\prime}\right)^{l_{1}}\left(i v_{2}^{\prime}\right)^{l_{2}} \cdots\left(i v_{b}^{\prime}\right)^{l_{b}} d v_{1}^{\prime} d v_{2}^{\prime} \cdots d v_{b}^{\prime} \\
= & -\int_{\mathbb{R}^{b}} e^{-\frac{1}{2} \beta^{\prime \prime}(\xi)\left(v^{\prime}, v^{\prime}\right)} s_{j}^{(k)}\left(\alpha, i v^{\prime}\right) d v^{\prime} .
\end{aligned}
$$

Thus

$$
\int_{\mathbb{R}^{b}} e^{-\frac{1}{2} \beta^{\prime \prime}(\xi)(v, v)} s_{j}^{(k)}(\alpha, i v) d v=0 .
$$

By Lemma 4, for $k$ odd the coefficients of $T^{-\frac{k}{2}}$ vanish. So we only need to calculate the coefficients when $k$ is even. Let $b_{j}^{(k)}(\alpha)$ be the coefficient of $T^{k}$ in 
Proposition 4. If we write $\alpha=\left(\alpha_{1}, \alpha_{2}, \ldots, \alpha_{b}\right)$ then

$$
\begin{aligned}
b_{j}^{(k)}(\alpha) & =\int_{\mathbb{R}^{b}} e^{-\frac{1}{2} \beta^{\prime \prime}(\xi)(v, v)} s_{j}^{(2 k)}(\alpha, i v) d v \\
& =\int_{\mathbb{R}^{b}} e^{-\frac{1}{2} \beta^{\prime \prime}(\xi)(v, v)} \sum_{l=0}^{2 k}(-1)^{l} \frac{f_{j}^{(l)}(i v)\langle\alpha, 2 \pi i v\rangle^{2 k-l}}{(2 k-l) !} d v \\
& =\sum_{l_{1}+l_{2}+\cdots+l_{b}=0}^{2 k} b_{l_{1} l_{2} \ldots l_{b}}^{(j)} \alpha_{1}^{l_{1}} \alpha_{2}^{l_{2}} \cdots \alpha_{b}^{l_{b}},
\end{aligned}
$$

where $b_{l_{1} l_{2} \cdots l_{b}}^{(j)}$ are constants. More precisely,

$$
\begin{aligned}
b_{l_{1} l_{2} \ldots l_{b}}^{(j)}= & \frac{\left(l_{1}+l_{2}+\cdots+l_{b}\right) !}{l_{1} ! l_{2} ! \ldots l_{b} !} \int_{\mathbb{R}^{b}} e^{-\frac{1}{2} \beta^{\prime \prime}(\xi)(v, v)}(2 \pi i)^{l_{1}+l_{2}+\cdots+l_{b}} \\
& \times v_{1}^{l_{1}} v_{2}^{l_{2}} \ldots v_{b}^{l_{b}} f_{j}^{\left(2 k-\left(l_{1}+l_{2}+\cdots+l_{b}\right)\right)}(i v) d v .
\end{aligned}
$$

Thus we have proved the following proposition.

Proposition 5.

$$
\begin{aligned}
& \left|\sum_{k=0}^{2 N+1} \frac{1}{T^{k / 2}} \int_{\|v\| \leqslant \sqrt{T} \rho} e^{-\frac{1}{2} \beta^{\prime \prime}(\xi)(v, v)} e^{-2 \pi i\langle\alpha, v / \sqrt{T}\rangle} f_{j}^{(k)}(i v) d v-\sum_{k=0}^{N} \frac{b_{j}^{(k)}(\alpha)}{T^{k}}\right| \\
& \quad \leqslant c \sup _{n \leqslant 4 N+b+2}\left\|y^{n} g\right\|_{L^{1}}\left(T^{-(N+1)}+e^{-\epsilon^{\prime} T}\right),
\end{aligned}
$$

where $b_{j}^{(k)}(\alpha)=\sum_{l_{1}+l_{2}+\ldots+l_{b}=0}^{2 k} b_{l_{1} l_{2} \ldots l_{b}}^{(j)} \alpha_{1}^{l_{1}} \alpha_{2}^{l_{2}} \cdots \alpha_{b}^{l_{b}}$ are polynomials in $\alpha_{1}, \alpha_{2}, \ldots, \alpha_{b}$ and the degree of $b_{j}^{(k)}$ is $2 k$ and $b_{l_{1} l_{2} \cdots l_{b}}^{(j)}$ are constants which depend on $g$.

Let

$$
\begin{gathered}
c_{n}(\alpha)=\sum_{i=0}^{n} b_{i}^{(n-i)}(\alpha)=\sum_{i=0}^{n}\left(\sum_{l_{1}+l_{2}+\cdots+l_{b}=0}^{2(n-i)} b_{l_{1} l_{2} \ldots l_{b}}^{(j)} \alpha_{1}^{l_{1}} \alpha_{2}^{l_{2}} \cdots \alpha_{b}^{l_{b}}\right) \\
:=\sum_{l_{1}+l_{2}+\cdots+l_{b}=0}^{2 n} c_{l_{1} l_{2} \cdots l_{b}} \alpha_{1}^{l_{1}} \alpha_{2}^{l_{2}} \cdots \alpha_{b}^{l_{b}},
\end{gathered}
$$

where $c_{l_{1} l_{2} \ldots l_{b}}$ are constants and

$$
c_{0}(\alpha)=b_{0}^{(0)}=f_{0}^{(0)} \int_{\mathbb{R}^{b}} e^{-\frac{1}{2} \beta^{\prime \prime}(\xi)(v, v)} d v=\frac{(2 \pi)^{b / 2}}{\sqrt{\left|\operatorname{det} \beta^{\prime \prime}(\xi)\right|}} \hat{g}\left(-i h^{*}\right)>0,
$$

so that $c_{0}(\alpha)$ is independent of $\alpha$. On the other hand, for $n \geqslant 1, c_{n}(\alpha)$ is polynomial in $\alpha_{1}, \ldots, \alpha_{b}$ whose degree is $2 n$ by Proposition 5 . From the expression of $b_{j}^{(k)}(\alpha)$, we have

$$
\begin{aligned}
c_{n}(\alpha) & \sim \int_{\mathbb{R}^{b}} e^{-\frac{1}{2} \beta^{\prime \prime}(\xi)(v, v)} f_{0}^{(0)}(i v) \frac{\langle\alpha, 2 \pi i v\rangle^{2 n}}{(2 n)} d v \\
& =(2 \pi i)^{2 n} \frac{f_{0}^{(0)}}{(2 n) !} \int_{\mathbb{R}^{b}} e^{-\frac{1}{2} \beta^{\prime \prime}(\xi)(v, v)}\langle v, \alpha\rangle^{2 n} d v \\
& \sim(-1)^{n} c_{g}\|\alpha\|^{2 n} \quad\left(c_{g}>0 \text { and } c_{g} \text { is dependent on } g\right),
\end{aligned}
$$


where $A \sim B$ means that $\lim _{\|\alpha\| \rightarrow \infty} A / B=1$. In order to make the result positive, we assume that $K<\frac{1}{\rho}$ so that

$$
e^{-\frac{c T}{T^{K} \rho}} \leqslant c \frac{1}{T^{N}}
$$

for any $N>0$. Now we obtain the following theorem.

Theorem 1. Let $M$ be a compact manifold with first Betti number $b>0$ and let $\phi_{t}$ : $M \rightarrow M$ be a homologically full transitive Anosov flow. Let $g$ be of class $C^{\infty}$ with compact support and $g \geqslant 0$. There exist $\xi \in \mathbb{R}^{b}, h^{*}>0$ such that for $\alpha \in H_{1}(M, \mathbb{Z})$, we have

$$
\pi_{g}(T, \alpha)=\frac{e^{T h^{*}}}{T^{b / 2+1}} e^{-\langle\xi, \alpha\rangle}\left(\sum_{n=0}^{N} \frac{c_{n, g}(\alpha)}{T^{n}}+O\left(\frac{1}{T^{N+1}}\right)\right) \quad \text { as } T \rightarrow \infty,
$$

for any $N \in \mathbb{N}$. If we write $\alpha=\left(\alpha_{1}, \alpha_{2}, \ldots, \alpha_{b}\right)$ then the constants $c_{n, g}(\alpha)$ are in the form

$$
c_{n, g}(\alpha)=\sum_{l_{1}+l_{2}+\cdots+l_{b}=0}^{2 n} c_{l_{1} l_{2} \ldots l_{d}} \alpha_{1}^{l_{1}} \alpha_{2}^{l_{2}} \cdots \alpha_{b}^{l_{b}},
$$

where $c_{l_{1}, l_{2}, \ldots, l_{b}}$ are constants which are independent of $\alpha$.

Remark. If $g$ is of class $C^{m}$ with compact support and $m$ satisfies $(m-\beta) / \rho \geqslant N+$ $\frac{b}{2}+2$ for some $N \in \mathbb{N}$, (9) still holds.

\section{The proof of the main result}

In this section we use an approximation argument to obtain $\pi(T, \alpha)$. Let $g$ be a characteristic function. For all $T$ we take $g_{T}^{-}$and $g_{T}^{+}$of class $C^{\infty}$ with compact supports such that:

(1) $g_{T}^{-} \leqslant g \leqslant g_{T}^{+}$;

(2) $\left\|g_{T}^{+}\right\|_{\infty} \leqslant 2$ and $\left\|g_{T}^{-}\right\|_{\infty} \leqslant 2$;

(3) for $0 \leqslant n \leqslant M,\left\|y^{n} g_{T}^{ \pm}\right\|_{L^{1}} \leqslant c\left\|y^{n} g\right\|_{L^{1}}$;

(4) $\sup _{n \leqslant M}\left\|y^{n}\left(g_{T}^{ \pm}-g\right)\right\|_{L^{1}} \leqslant T^{-\lambda} \sup _{n \leqslant M}\left\|y^{n} g\right\|_{L^{1}}$ for some $\lambda>0$.

(5) $\left\|g_{T}^{ \pm(m)}\right\|_{L^{1}} \leqslant c T^{m \lambda}\|g\|_{L^{1}}$.

These can be done by a convolution argument.

By (7), we have that

$$
\begin{aligned}
& \left|\pi_{g_{T}^{ \pm}}(T, \alpha)-e^{-\langle\xi, \alpha\rangle} \sum_{j=0}^{M} \frac{1}{T^{j+1}} \int_{V_{0}} e^{-2 \pi i\langle v, \alpha\rangle} \frac{d^{j} \widehat{g_{T}^{ \pm}}}{d s^{j}}(-i \beta(\xi+i v)) e^{\beta(\xi+i v) T} d v\right| \\
& \quad \leqslant \frac{c\left\|y^{M+1} g_{T}^{ \pm}\right\|_{L^{1}}}{T^{M+1}} e^{T h^{*}}+c \frac{\| g_{T}^{ \pm}}{T^{(m-\beta)} \|_{L^{1}}} e^{T h^{*}}+c\left(\left\|g_{T}^{ \pm}\right\|_{L^{1}}+\cdots+\left\|y^{M} g_{T}^{ \pm}\right\|_{L^{1}}\right) e^{T h^{*}-\frac{c T}{T^{K} \rho}} \\
& \quad \leqslant c_{0} \frac{\left\|y^{M+1} g\right\|_{L^{1}}}{T^{M+1}} e^{T h^{*}}+c_{1} \frac{\|g\|_{L^{1}}}{T^{(m-\beta) K-m \lambda}} e^{T h^{*}}+c_{2}\left(\|g\|_{L^{1}}+\cdots+\left\|y^{M} g\right\|_{L^{1}}\right) e^{T h^{*}-\frac{c T}{T^{K} \rho}} .
\end{aligned}
$$

By condition (4),

$$
\begin{aligned}
& \sum_{j=0}^{M} \frac{1}{T^{j+1}}\left|\int_{V_{0}} e^{-2 \pi i\langle v, \alpha\rangle}\left(\frac{d^{j} \widehat{g_{T}^{+}}}{d s^{j}}-\frac{d^{j} \widehat{g}}{d s^{j}}\right)(-i \beta(\xi+i v)) e^{\beta(\xi+i v) T} d v\right| \\
& \quad \leqslant c_{3} \sup _{n \leqslant M}\left\|y^{n} g\right\|_{L^{1}} \frac{e^{T h^{*}}}{T^{\frac{b}{2}+1}} \frac{1}{T^{\lambda}} .
\end{aligned}
$$


Hence,

$$
\begin{aligned}
& \pi_{g}(T, \alpha)-e^{-\langle\xi, \alpha\rangle} \sum_{j=0}^{M} \frac{1}{T^{j+1}} \int_{V_{0}} e^{-2 \pi i\langle v, \alpha\rangle} \frac{d^{j} \widehat{g}}{d s^{j}}(-i \beta(\xi+i v)) e^{\beta(\xi+i v) T} d v \\
& \leqslant \pi_{g_{T}^{+}}(T, \alpha)-e^{-\langle\xi, \alpha\rangle} \sum_{j=0}^{M} \frac{1}{T^{j+1}} \int_{V_{0}} e^{-2 \pi i\langle v, \alpha\rangle} \frac{d^{j} \widehat{g}}{d s^{j}}(-i \beta(\xi+i v)) e^{\beta(\xi+i v) T} d v \\
& \leqslant \pi_{g_{T}^{+}}(T, \alpha)-e^{-\langle\xi, \alpha\rangle} \sum_{j=0}^{M} \frac{1}{T^{j+1}} \int_{V_{0}} e^{-2 \pi i\langle v, \alpha\rangle} \frac{d^{j} \widehat{g_{T}^{+}}}{d s^{j}}(-i \beta(\xi+i v)) e^{\beta(\xi+i v) T} d v \\
& \quad+e^{-\langle\xi, \alpha\rangle} \sum_{j=0}^{M} \frac{1}{T^{j+1}}\left|\int_{V_{0}} e^{-2 \pi i\langle v, \alpha\rangle}\left(\frac{d^{j} \widehat{g_{T}^{+}}}{d s^{j}}-\frac{d^{j} \widehat{g}}{d s^{j}}\right)(-i \beta(\xi+i v)) e^{\beta(\xi+i v) T} d v\right| \\
& \leqslant c_{0} \frac{\left\|y^{M+1} g\right\|_{L^{1}}}{T^{M+1}} e^{T h^{*}}+c_{1} \frac{\|g\|_{L^{1}}}{T^{(m-\beta) K-m \lambda}} e^{T h^{*}} \\
&+c_{2}\left(\|g\|_{L^{1}}+\cdots+\left\|y^{M} g\right\|_{L^{1}}\right) e^{T h^{*}-\frac{c T}{T^{K} \rho}}+c_{3} \sup _{n \leqslant M}\left\|y^{n} g\right\|_{L^{1}} \frac{e^{T h^{*}}}{T^{\frac{b}{2}+1}} \frac{1}{T^{\lambda}} .
\end{aligned}
$$

Taking $M \geqslant \frac{b}{2}+1+\lambda$ and $g=\chi_{\left[-T^{1 / 4 M+2}, 0\right]}$, then

$$
\begin{gathered}
\left|\pi_{g}(T, \alpha)-e^{-\langle\xi, \alpha\rangle} \sum_{j=0}^{M} \frac{1}{T^{j+1}} \int_{V_{0}} e^{-2 \pi i\langle v, \alpha\rangle} \frac{d^{j} \widehat{g}}{d s^{j}}(-i \beta(\xi+i v)) e^{\beta(\xi+i v) T} d v\right| \\
\leqslant e^{T h^{*}}\left(\frac{c_{0}}{T^{M}}+\frac{c_{1}}{T^{(m-\beta) K-m \lambda-1}}+c_{2} e^{-\frac{c T}{T^{K} \rho}}+\frac{c_{3}}{T^{\frac{b}{2}+\lambda}}\right) .
\end{gathered}
$$

Let $\zeta=\min \left\{\lambda-1,(m-\beta) K-m \lambda-\frac{b}{2}-2\right\}$, where $K<1 / \rho$. Since we can take $M$ sufficiently large, the best error term is $1 / T^{\zeta}$. By $\lambda-1>0$ and $(m-\beta) K-m \lambda-$ $\frac{b}{2}-2>0$, we have $\lambda<K<1 / \rho$ if we let $m \rightarrow \infty$. We assume that $\rho<1$ and let $\delta=\left[\frac{1}{\rho}\right]-1$. We do the same as was done in the last section for

$$
\int_{V_{0}} e^{-2 \pi i\langle v, \alpha\rangle}\left(d^{j} \widehat{g} / d s^{j}\right)(-i \beta(\xi+i v)) e^{\beta(\xi+i v) T} d v
$$

and obtain

$$
\begin{gathered}
\sum_{j=0}^{M} \frac{1}{T^{j+1}} \int_{V_{0}} e^{-2 \pi i\langle v, \alpha\rangle} \frac{d^{j} \widehat{g}}{d s^{j}}(-i \beta(\xi+i v)) e^{\beta(\xi+i v) T} d v \\
=\frac{e^{T h^{*}}}{T^{\frac{b}{2}+1}}\left(c_{0}+\sum_{k=1}^{N} \frac{c_{k, T}(\alpha)}{T^{k}}+O\left(\frac{1}{T^{N}}\right)\right) .
\end{gathered}
$$

Since for $g=\chi_{\left[-T^{\frac{1}{4 M+2}}, 0\right]}, \hat{g}(-i \beta(\xi+i v))=g(\xi+i v)+O\left(e^{-T^{\frac{1}{4 M+2}} h^{*}}\right)$, hence

$$
c_{k, T}(\alpha)=c_{k}(\alpha)+O\left(e^{T h^{*}-T \frac{1}{4 M+2}} h^{*}\right) .
$$

Hence

$$
\pi_{g}(T, \alpha)=\frac{e^{T h^{*}}}{T^{b / 2+1}}\left(c_{0}+\sum_{k=1}^{N} \frac{c_{k}(\alpha)}{T^{k}}+O\left(\frac{1}{T^{N}}\right)\right)
$$


It is well known that

$$
\pi\left(T-T^{\frac{1}{4 M+2}}, \alpha\right)=O\left(e^{T h^{*}-T^{\frac{1}{4 M+2}}} h^{*}\right)
$$

and

$$
\pi\left(T-T^{\frac{1}{4 M+2}}, \alpha\right)+\pi_{\chi_{\left[-T \frac{1}{4 M+2}, 0\right]}}(T, \alpha)=\pi(T, \alpha) .
$$

However, $e^{-h^{*} T^{1 / 4 M+2}} \rightarrow 0$ faster than $1 / T^{n}$ for any $n$. We have the following theorem.

Theorem 2. Let $M$ be a compact manifold with first Betti number $b>0$ and let $\phi_{t}: M \rightarrow M$ be a homologically full transitive Anosov flow. There exist $\xi \in \mathbb{R}^{b}, h^{*}>0$ and $\delta>0$ such that for $\alpha \in H_{1}(M, \mathbb{Z})$, we have

$$
\pi(T, \alpha)=\frac{e^{T h^{*}}}{T^{b / 2+1}} e^{-\langle\xi, \alpha\rangle}\left(c_{0}+\sum_{n=1}^{N} \frac{c_{n}(\alpha)}{T^{n}}+O\left(\frac{1}{T^{\delta}}\right)\right) \quad \text { as } T \rightarrow \infty
$$

for $N<\delta$, where $c_{0}>0$ is a constant which is independent of $\alpha$. If we write $\alpha=$ $\left(\alpha_{1}, \alpha_{2}, \ldots, \alpha_{b}\right)$ then the constants $c_{n}(\alpha)$ are in the form

$$
c_{n}(\alpha)=\sum_{l_{1}+l_{2}+\cdots+l_{b}=0}^{2 n} c_{l_{1} l_{2} \ldots l_{d}} \alpha_{1}^{l_{1}} \alpha_{2}^{l_{2}} \cdots \alpha_{b}^{l_{b}},
$$

where $c_{l_{1}, l_{2}, \ldots, l_{b}}$ are constants which are independent of $\alpha$.

Remark. As we see in [5] for closed geodesics, we still have that

$$
c_{n}(\alpha) \sim(-1)^{n} c\|\alpha\|^{2 n} \quad(c>0) \text { as }\|\alpha\| \rightarrow \infty .
$$

Acknowledgements. (1) After obtaining the results of this paper, the author learned that Motoko Kotani [4] had studied the same question for closed geodesics and also obtained how the coefficients $c_{n}(\alpha)$ of the error terms depend on the homology class $\alpha$ for geodesic flows.

(2) The author would like to thank Richard Sharp, for his suggestion of the field of study, fruitful discussions and encouragement, and Mark Pollicott for his advice and encouragement. The author also wishes to thank CVCP and Mathematics Department of Manchester University for their financial support.

\section{REFERENCES}

[1] N. Anantharaman. Precise counting results for closed orbits of Anosov flows. Ann. Sci. Ećole Norm. Sup. (4) 33 (2000), 33-56.

[2] R. Bowen. Symbolic dynamics for hyperbolic flows. Amer. J. Math. 95 (1973), 429-459.

[3] D. Dolgopyat. Prevalence of rapid mixing in hyperbolic flows. Ergodic Theory and Dynam. Sys. 18 (1998), 1097-1114.

[4] M. Kotani. A note on asymptotic expansions for closed geodesics in homology classes. Math. Ann. (3) 320 (2001), 507-529.

[5] D. Liu. Ph.D Thesis.

[6] A. Manning. Axiom A diffeomorphisms have rational zeta functions. Bull. London Math. Soc. 3 (1971), 215-220.

[7] W. Parry and M. Pollicott. Zeta functions and the periodic orbit structure of hyperbolic dynamics. Astérisque (1990) 187-188. 
[8] M. Pollicott and R. Sharp. Asymptotic expansions for closed orbits in homology classes. Geom. Dedicata. 87 (2001), 123-160.

[9] M. Pollicott and R. Sharp. Error terms for closed orbits of hyperbolic flows. Ergodic Theory Dynam. Systems 21 (2001), 545-562.

[10] D. RuElle. An extension of the theory of Fredholm determinants. Inst. Hautes Études Sci. Publ. Math. 72 (1990), 175-193.

[11] R. Sharp. Closed orbits in homology classes for Anosov flows. Ergodic Theory Dynam. Systems. 13 (1993), 387-408. 